formed a normal distribution curve, with the 97.5th centile falling at $16.8 \mathrm{mIU} / \mathrm{L}$. Therefore, cord TSH exceeding 16.8 $\mathrm{mIU} / \mathrm{L}$ formed the basis for screening cut-off TSH values at SGH since 2013.

Objectives The objective of our study was to determine the prevalence of $\mathrm{CH}$ amongst newborn infants born at SGH who had umbilical cord TSH levels in the borderline range 16.8 $25.0 \mathrm{mIU} / \mathrm{L}$.

Methods In this retrospective cohort study, livebirths from 1 January 2015 to 31 October 2020 who had borderline cord TSH levels of $16.8-25.0 \mathrm{mIU} /$ Lwere included. Neonates with raised cord TSH $\geq 16.8 \mathrm{mIU} / \mathrm{L}$ had thyroid function repeated on day 6-8 and 2-weekly thereafter. Demographic and birth data were collected, including cord free thyroxine (fT4) and TSH levels, aetiology, age at initiation of treatment, initial dose and length of outpatient follow-up.

Results Our screening program based on cord TSH cut-off $\geq 16.8 \mathrm{mIU} / \mathrm{L}$ resulted in a positive screening rate of $3.63 \%$. A total of 276 of 10,172 neonates $(2.71 \%)$ born during the study period was found to have cord TSH levels in the range $16.8-25.0 \mathrm{mIU} / \mathrm{L}$. The mean ( \pm S.D.) gestational age was 38.2 $( \pm 1.9)$ weeks and mean $( \pm$ S.D.) birth weight was 3040 $( \pm 486)$ grams. There were three cases of congenital hypothyroidism amongst 276 livebirths, giving a prevalence rate of 1 in 92. All 3 neonates were born at term with mean birth weight 3,287 grams at a mean gestational age 38 weeks. Of the 3 , one was diagnosed postnatally with trisomy 21 complicated by patent ductus and ventricular septal defect, while another was born to a mother with Graves' disease. All had thyroid dysgenesis and were given daily L-thyroxine replacement.

Conclusions Our current newborn screening program for congenital hypothyroidism based on an umbilical cord TSH cut-off $\geq 16.8 \mathrm{mIU} / \mathrm{L}$ resulted in a positive screening rate of $3.63 \%$. Amongst 276 neonates with TSH levels of 16.8 - $25.0 \mathrm{mIU} / \mathrm{L}$, three term infants were diagnosed with congenital hypothyroidism, giving a prevalence rate of 1 in 92. These 3 cases of $\mathrm{CH}$ would have been missed if the screening cut-off level were increased to $25 \mathrm{mIU} / \mathrm{L}$. A better screening TSH cut-off at the 97.5 th centile of the distribution curve appeared to be $18 \mathrm{mIU} / \mathrm{L}$ instead of 16.8 $\mathrm{mIU} / \mathrm{L}$.

\section{CASE OF INFANT BOTULISM AND REVIEW OF THE USE OF HEPTAVALENT BOTULINUM ANTITOXIN FOR TREATMENT}

Karen Donceras Nadua, Ann Chuan En Loh, Terrence Gerard Sundram Thomas, Matthias Maiwald, Xue Fen Valerie Seah, Natalie Woon Hui Tan. Singapore

\subsection{6/bmjpo-2021-RCPCH.193}

Background Infant botulism is a rare and potentially lethal condition resulting in symmetrical descending flaccid paralysis, caused by ingestion of Clostridium botulinum spores. Human Botulism Immune Globulin Intravenous (BIG-IV) is deemed safe and effective in reducing inpatient and intensive care unit (ICU) stay for infants with botulism. However, the high cost of BIG-IV and the time to procurement from the United States can be prohibitive. A possible alternative is the heptavalent botulinum antitoxin (HBAT), an equine-derived antitoxin licensed for use for treatment of non-infant botulism.
Objectives To present a case of infant botulism and review the use and safety of heptavalent botulinum antitoxin as treatment for infant botulism.

Methods Case report and review of literature.

Results A three-month-old female infant weighing $5.3 \mathrm{~kg}$ presented to our hospital with two days of reduced feeding, poor suck and lethargy. She subsequently developed acute flaccid paralysis with bulbar involvement. History revealed ingestion of 1 milliliter of raw honey about 3 weeks prior to symptom onset. She was transferred to the ICU for non-invasive respiratory support on and also required nasogastric tube feeding. Her stool botulism neurotoxin gene PCR was positive for type A and B genes. A nerve conduction study demonstrated a pre-synaptic neuromuscular junction defect consistent with the diagnosis of infant botulism.

A collaborative decision between parents and clinicians was made for HBAT over BIG-IV due to lower cost and availability in Singapore. On day 6 of admission, she was given HBAT (10\% of vial, $1.6 \mathrm{~mL}$ once) using a slow incremental infusion rate, with close monitoring of vital signs and with diphenhydramine as premedication. She did not experience any adverse effects. Her condition improved and she was eventually discharged after 18 days of hospitalization.

Only few studies describe the use of HBAT in infant botulism and the experience in children is limited, especially in infants. This adds to the limited data available for the use of HBAT in infants, and suggests that HBAT may be safe and effective for use in infant botulism.

Conclusions Infant botulism is a rare condition that requires prompt diagnosis and treatment. In a setting where the BIGIV is not accessible in a timely manner due to high cost or logistics, HBAT can be considered as a safe and effective alternative for the management of infant botulism.

\section{LOPINAVIR/RITONAVIR TREATMENT IN PAEDIATRIC PATIENTS WITH COVID-19}

Po Ki Ho, JSC Wong, Kk To, P Ip, Ys Yau, Yw Kwan. Hong Kong

\subsection{6/bmjpo-2021-RCPCH.194}

Background Protease inhibitor lopinavir/ritonavir has been used alone or in combination with other therapeutics as treatment of COVID-19. Data on paediatric population are scarce. Objectives To assess efficacy and safety of lopinavir/ritonavir for treating paediatric patients with COVID-19.

Methods From March to August 2020, patients aged 18 years or below with confirmed COVID-19 diagnosed by positive SARS-CoV-2 reverse transcriptase-polymerase chain reaction (RT-PCR) in their respiratory specimens in Department of Paediatrics, Queen Elizabeth Hospital and Princess Margaret Hospital, Hong Kong, were included. Patients were treated with lopinavir/ritonavir at decision of primary physician for 14 days or shorter when patient was fit for discharge or PCR in nasopharyngeal swab (NPS) turned negative. NPS viral load, which was determined by RT-qPCR targeting the RdRp-Hel gene, was obtained at different study time points. Clinical features including demographics, duration of symptom and hospitalization, time to negative PCR in NPS, need of oxygen therapy or intensive care were retrieved from medical records.

Results 80 patients admitted from March 2020 to August 2020 for COVID-19 were recruited. 27(33.8\%) received 
lopinavir/ritonavir and 53(66.2\%) patients received standard of care. Median age of patients in both groups were 8 years. Most patients have no co-morbidity. 16(59.3\%), 8(29.6\%) and $3(11.1 \%)$ patients in treatment group while 33(62.3\%), 20 $(37.7 \%)$ and none of patients in control group have upper respiratory infections, asymptomatic infection and pneumonia respectively. The median days from symptom onset to admission was 1 (IQR 0-2) in treatment and 3(IQR 1-5) in control group. The median days from symptom onset to start of lopinavir/ritonavir was $1(\mathrm{IQR} 1-3)$. The NPS viral load reduction from baseline to day 7 was greater in treatment group. Viral load dropped from mean $6.5 \log ^{10}$ copies/ml (SD 2.1) on admission to mean $4.6 \log ^{10}$ copies/ml (SD 2.1) on day 7 in patients treated with lopinavir/ritonavir. Viral load dropped from mean $6.4 \log ^{10}$ copies/ml (SD 1.8) on admission to mean $5.3 \log ^{10}$ copies/ml (SD 1.5) on day 7 in patient who did not receive lopinavir/ritonavir. However, the difference was not statistically significant. There was no significant difference in viral load on day 10 and day 14 between the two groups. There was also no significant difference in time to symptom resolution, PCR negativity or duration of hospitalization. No patient in the cohort require oxygen or intensive care. Among those received treatment, 7(25.9\%) patients experienced gastrointestinal symptoms possibly drug adverse effects with self-limited nausea, vomiting or diarrhea, as compared to $9(17 \%)$ patients in control group. 1(3.7\%) and $3(5.7 \%)$ patients in treatment and control group have mild deranged liver function. There was no significant difference in rate of gastrointestinal symptom or liver function derangement among the two groups. There was no QT interval prolongation or serious adverse effect noted in patients received treatment.

Conclusions There was no statistically significant difference in NPS viral load clearance, time to symptom resolution, PCR negativity and duration of hospitalization between paediatric COVID-19 patients treated with lopinavir/ritonavir and control. Self-limited gastrointestinal symptoms were common but use of lopinavir/ritonavir appeared to be safe in children with no serious side effect noted.

\section{PSYCHOLOGICAL ILL-EFFECTS AND RESILIENCE OF PROVIDERS OF CHILDREN WITH DEVELOPMENTAL DISABILITIES DURING THE COVID-19 PANDEMIC}

Ramkumar Aishworiya, Tammy SH Lim, Ying Qi Kang, Phyllis PL Lim, Shang Chee Chong, Mae Yue Tan. Singapore

\subsection{6/bmjpo-2021-RCPCH.195}

Background Providers of children with developmental disabilities (DD) had to rapidly innovate to translate face to face care to a virtual platform in order to continue to support the needs of their patients during the coronavirus 2019 pandemic. Objectives We aimed to explore the extent of psychological ill-effects, if any, and explore resilience in providers of children with DD.

Methods This was a voluntary cross-sectional study involving an anonymous questionnaire, administered via a secure online platform, between 13 May and 1 July 2020, coinciding with the Circuit Breaker (similar to a lockdown) period in Singapore. Participants included healthcare providers serving children with DD in various capacities at public and private healthcare settings in the country. Common diagnoses of DD included autism spectrum disorder and global developmental

\begin{tabular}{|c|c|c|c|c|c|c|}
\hline Variable & $\begin{array}{l}\text { Depression } \\
\text { (N) }\end{array}$ & $\%$ & $\begin{array}{l}\text { Anxiety } \\
\text { (N) }\end{array}$ & $\%$ & $\begin{array}{l}\text { Stress } \\
\text { (N) }\end{array}$ & $\%$ \\
\hline $\begin{array}{l}\text { Prevalence of positive screen } \\
\text { of traits on the DASS }\end{array}$ & 13 & 13.7 & 40 & 42.1 & 54 & 56.8 \\
\hline \multicolumn{7}{|c|}{ Among those who screened positive } \\
\hline Mild & 7 & 53.8 & 6 & 15 & 8 & 14.8 \\
\hline Moderate & 3 & 23.1 & 10 & 25 & 8 & 14.8 \\
\hline Severe & 1 & 7.7 & 6 & 15 & 9 & 16.7 \\
\hline Extremely Severe & 2 & 15.4 & 18 & 45 & 29 & 53.7 \\
\hline
\end{tabular}

delay. The Depression, Anxiety, and Stress Scales (DASS-21) and Connor-Davidson Resilience Scale 25 -item were administered. Descriptive statistics were conducted.

Results Ninety-five responses were received. Mean age of respondents was 37.4 years (SD 9.45), with $96.8 \%$ of them being female. A quarter (24.5\%) were medical professionals (doctor, nurse, psychologists), a third (33.0\%) were therapists (physiotherapists, speech therapists and occupational therapists), a third were school-based providers (teachers in mainstream or special-education schools, learning support educators), and the rest (8.5\%) were working in administrative aspects of dealing with children with DD (case manager, social workers, administrative staff). A quarter (25.5\%) worked in a hospital, 20.2\% worked in a school setting, $37.2 \%$ at a therapy centre (e.g early intervention centres), while $17.0 \%$ worked in social community agencies.

On the DASS (table 1), more than half $(56.8 \%$ ) of the respondents had a positive screen for stress, of which half of these (53.7\%) had levels which were in the 'extremely severe' range. Almost half (42.1\%) of providers had anxiety, of which $45.0 \%$ of these were 'extremely severe'. Depression rate was lower, with a positive screen present in $13.7 \%$ in this group of providers, and half of these were in the 'mild' range. Chi-square analysis showed that providers in schoolbased institutions were more likely than providers in nonschool-based institutions to have a positive screen for depression on the DASS-21 (36.8\% vs $8.0 \%, p=0.004)$. Mean resilience scores on the CD-RISC 25 was 62.7 (range 36.0 to 88.0 ), lower compared to the published normative data for this scale.

Conclusions Providers of children with DD face significant psychological ill-effects during this pandemic, as well as lower resilience compared to the general population. Targeted institutional support for these providers and interventions to improve their resilience are both crucial to enhance the mental well-being of these providers. This will in turn facilitate continued provision of care for children with DD despite the pandemic.

\section{OUTCOME OF FLASH GLUCOSE MONITORING- AN AUDIT}

Nikitha Rajaraman, Fathima Haniffa, Dhaara lyer. UK

\subsection{6/bmjpo-2021-RCPCH.196}

Background The use Flash Glucose Monitoring (FGM) is considered a useful technology for self-management of type 1 diabetes mellitus (T1DM). 